\title{
Isolation, Purification, and Identification of a Novel HX-Producing Strain and Optimization of Its Fermentation Medium
}

\author{
Zhengkai Xue ${ }^{1}$, Wenwu Zheng ${ }^{2 *}$ \\ ${ }^{1}$ School of White Liquor, Luzhou Vocational and Technical College, Luzhou, China \\ ${ }^{2}$ Department of Cardiology, The Affiliated Hospital of Southwest Medical University, Luzhou, China \\ Email: *zhengwenwu888@163.com
}

How to cite this paper: Xue, Z.K. and Zheng, W.W. (2018) Isolation, Purification, and Identification of a Novel HX-Producing Strain and Optimization of Its Fermentation Medium. Food and Nutrition Sciences, 9, 447-463. https://doi.org/10.4236/fns.2018.95035

Received: February 27, 2018

Accepted: May 14, 2018

Published: May 17, 2018

Copyright (C) 2018 by authors and Scientific Research Publishing Inc. This work is licensed under the Creative Commons Attribution International License (CC BY 4.0).

http://creativecommons.org/licenses/by/4.0/

\section{c) (i) Open Access}

\begin{abstract}
Hexanoic acid (HX) is a crucial flavor compound and precursor of ethyl caproate (EA), which determines the quality of Chinese Luzhou-flavor liquor (CLFL). The isolation, purification, identification, and optimization of fermentation conditions of HX-producing bacteria are essential for industrial CLFL production. In this study, one strain of HX-producing bacterium was isolated from six candidate bacterial strains and identified as Clostridium sartagoneforme. Then, the growth characteristics and HX production of $C$. sartagoneforme were investigated. Sodium acetate medium was identified as the optimal fermentation medium from four candidate media. C. sartagoneforme yielded $800.85 \pm 12.87 \mathrm{mg} / 100 \mathrm{~mL} \mathrm{HX}$ in sodium acetate medium. Then, to further optimize the formula of the fermentation medium, the carbon and nitrogen sources and inorganic salt component of the fermentation medium were investigated using HX yields as an optimization index. Optimization was performed with a single-factor experiment and the Taguchi design method. The single-factor experiment showed that the highest HX outputs were obtained when the sodium acetate medium contained $2.5 \mathrm{~g} / \mathrm{L}$ yeast extract, 1.8 $\mathrm{g} / \mathrm{L} \mathrm{KCl}, 20 \mathrm{~g} / \mathrm{L}$ sodium acetate, $15 \mathrm{~mL} / \mathrm{L}$ ethanol, and $1.5 \mathrm{~g} / \mathrm{L}$ glucose. In the orthogonal experiment designed using the Taguchi design method, HX yields reached $2018.29 \pm 46.37 \mathrm{mg} / 100 \mathrm{~mL}$ in sodium acetate medium that contained $3.5 \mathrm{~g} / \mathrm{L}$ yeast extract, $1.8 \mathrm{~g} / \mathrm{L} \mathrm{KCl}, 25 \mathrm{~g} / \mathrm{L}$ sodium acetate, and $15 \mathrm{~mL} / \mathrm{L}$ ethanol.
\end{abstract}

\section{Keywords}

Isolation, Identification, Hexanoic Acid-Producing Strain, Fermentation Medium, Optimization 


\section{Introduction}

Hexanoic acid (HX) is the precursor of ethyl caproate (EA), the main flavor component in Chinese Luzhou-flavor liquor (CLFL) according to the National Standard GB/T 10781.1-2006. EA is produced during the late fermentation stage of CLFL production in a mud pit, a fermentation con tainer, by spirit reaction with HX. EA yields are determined by HX quantity from excessive spirits in lees, or fermented grains. The improvement of HX yields in CLFL production is a crucial technical problem for CLFL industries. Numerous methods have been developed to increase HX output in traditional liquor production [1] [2] [3].

One of the simplest and most widely adopted methods for improving CLFL quality is prolonged fermentation time. The fermentation time for regular liquor production is 45 days from lees entry to lees exit. To improve HX yield, the fermentation time for a pit in good fermentation condition is extended to 60,90 , or even 180 days. The relative HX amount, EA content, and CLFL quality increase as fermentation time increases. Another widely adopted method for improving CLFL production is multiple fermentation rounds (double fermentation). In this method, lees are retained in the bottom of the fermentation pit and are mixed with new fermentation starter powders (e.g., daqu in Chinese) for another one or two fermentation periods. After two or three fermentation periods, the amounts of HX and other organic acids increase and the microbial biochemical reaction of spirits with $\mathrm{HX}$ or other organic acids is enhanced, thus increasing total acid and ester contents and improving liquor flavor. The traditional methods to improve CLFL quality, however, decrease production efficiency and increase production cost [4]-[9].

For several centuries, the attempts of CLFL makers to economically improve liquor quality have been unsuccessful. A breakthrough in CLFL production finally occurred in the 1960s when EA was identified as the main flavor component of CLFL. The isolation, purification, and identification of HX-producing bacteria and the optimization of fermentation conditions are now routine work for the CLFL industry and researchers [10] [11] [12] [13].

In the past decades, several HX-producing microbes have been isolated and identified from CLFL pit mud. The HX production of these bacterial strains outside of fermentation pits, however, is limited at $700 \mathrm{mg} / \mathrm{mL}$ HX [12].

In this study, we aimed to isolate and identify highly effective HX-producing bacteria. We also aimed to optimize fermentation conditions for the application of HX-producing bacteria in practical production.

\section{Materials and Methods}

\subsection{Materials}

\subsubsection{Pit Mud}

The pit mud used in the present study was collected from the four corners and center of the bottom of a 180-year-old pit in Luzhou Laojiao Group Luzhou Laojiao Group Co. Ltd., a famous CLFL producer in Southern Sichuan, PRC. 


\subsubsection{Reagent}

Yeast extracts, $\mathrm{KCl}$, glucose, sodium acetate, and absolute ethanol were purchased from Kelong Reagent Factory (China, Chengdu). The standard material 2-ethyl butyrate was purchased from Aladdin Reagent Co., Ltd. (China, Shanghai).

\subsubsection{Culture Medium}

Isolation and enrichment culture medium:

The culture medium contained the following components: $5 \mathrm{~g}$ sodium acetate; 1 g yeast extracts; $10 \mathrm{~mL} 4 \%$ potassium dihydrogen phosphate; $5 \mathrm{~mL} 2 \%$ magne-

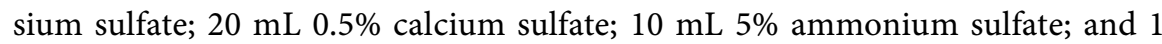
$\mathrm{mL} 5 \mathrm{mg} / \mathrm{L}$ biotin. The culture medium was brought to $1000 \mathrm{~mL}$ with tap water and its $\mathrm{pH}$ was adjusted to $6.0-6.5$. Then, $2 \%$ agar was added to solidify the medium before sterilization at $121^{\circ} \mathrm{C}$ for $30 \mathrm{~min}$ in an autoclave. Prior to inoculation, $20 \mathrm{~mL}$ absolute ethanol was added to the medium.

Fermentation medium:

Four fermentation media were selected for screening HX-producing bacteria (Xue et al., 2014).

\subsection{Methods}

\subsubsection{Isolation of HX-Producing Bacteria from Pit Mud}

Pit mud $\rightarrow$ Preparation of seed solution $\rightarrow$ Enrichment culture Plate separation $\rightarrow$ Slope inoculation $\rightarrow$ Primary screening $\rightarrow$ Rescreening $\rightarrow$ Gas chromatography (GC) analysis $\rightarrow$ Strain preservation.

\section{Preparation of Seed Solution}

A total of $1 \mathrm{~g}$ pit mud was added to $9 \mathrm{~mL}$ liquid isolation medium in a stoppered test tube. The tube was then placed in a $90^{\circ} \mathrm{C}$ water bath for $10 \mathrm{~min}$. After cooling, $150 \mu \mathrm{L}$ absolute ethanol was added to the tube. The seed solution was then cultured at $34^{\circ} \mathrm{C}$ and $0.08 \mathrm{MPa}$ in a vacuum incubator until gas was produced.

\section{Enrichment Culture}

A total of $1 \mathrm{~mL} \mathrm{HX-producing} \mathrm{seed} \mathrm{solution} \mathrm{was} \mathrm{pipetted} \mathrm{and} \mathrm{added} \mathrm{to} 9 \mathrm{~mL}$ liquid isolation culture medium in a stoppered tube. The tube was then placed in a $90^{\circ} \mathrm{C}$ water bath for $10 \mathrm{~min}$. After cooling to $70^{\circ} \mathrm{C}, 150 \mu \mathrm{L}$ absolute ethanol was added to the enrichment culture. The culture was then incubated under the above-mentioned conditions for 15 days.

\section{Plate Separation}

The tube of enrichment culture that produced the highest amount of gas was selected. A total of $10 \mu \mathrm{L}$ enriched culture medium was drawn from the selected tube, spread on solid culture medium, and incubated at $90^{\circ} \mathrm{C}$ in an incubator for $10 \mathrm{~min}$. Subsequently, $2 \%(\mathrm{~V} / \mathrm{V})$ absolute ethanol was added to the incubated culture medium until the culture medium cooled down to $70^{\circ} \mathrm{C}$. The inoculated medium was incubated under the above conditions until the colonies of HX-producing bacteria appeared. 


\section{Slant culture}

Colonies that possessed a regular morphology were selected and transferred to the surfaces of slant media and cultured in the above-mentioned conditions until the colonies of HX-producing bacteria appeared.

\section{Preliminary Screening}

Colonies with entire edges and slightly raised and smoothly rounded tops were selected. A loopful of bacteria was picked up, placed in a drop of water on a slide, and stained by Gram staining. The stained bacteria were observed under microscopy. Motile, Gram-positive, rod-shaped bacterial cells with ellipsoid spores were selected for future screening.

\section{Rescreening}

Individual colonies from six selected clones were picked and inoculated into 5 $\mathrm{mL}$ isolation culture medium under the above conditions for 6 days. The bacteria were then transferred to $50 \mathrm{~mL}$ of fermentation culture medium under the above conditions for 15 days. GC was used to investigate 15-day-old fermentation broths to select the most efficient strain of HX-producing bacteria based on HX yields.

\section{Pretreatment of fermented broth}

The $\mathrm{pH}$ of $1 \mathrm{~mL}$ fermented esterifying liquid was adjusted to $\mathrm{pH} 3$ with $1 \mathrm{M}$ hydrochloric acid solution. The amount of hydrochloric acid solution required to adjust the solution $\mathrm{pH}$ to 3 was recorded.

A total of $10 \mu \mathrm{L} 2$-ethyl butyrate, the internal standard solution, was added to $490 \mu \mathrm{L}$ fermented esterifying liquid. The mixture was vortexed vigorously. Then, the mixture was acidified to $\mathrm{pH} 3$ with $1 \mathrm{M}$ hydrochloric acid solution at half the volume of the $1 \mathrm{~mL}$ esterifying liquid. The acidified mixture was vortexed vigorously and then centrifuged for 10 minutes at $12,000 \mathrm{r} / \mathrm{min}$. The supernatant was drawn and filtered using a0.22- $\mu \mathrm{M}$ filter and stored at $4^{\circ} \mathrm{C}$ for future use.

\section{Conditions of GC analysis}

Chromatographic column: LZP-930 Liquor special analysis column (purchased from the Chinese Academy of Sciences, Lanzhou Institute of Chemical Physics).

Chromatographic conditions:

Detector temperature: $200^{\circ} \mathrm{C}$, Vaporizer temperature: $200^{\circ} \mathrm{C}$,

Injection mode: Split ratio: 50:1,

Column length: $25.0 \mathrm{~mm}$,

Inner diameter: $0.32 \mathrm{~mm}$,

Coating thickness: $1 \mu \mathrm{m}$,

Programmed temperature: $65^{\circ} \mathrm{C} \rightarrow 5 \mathrm{~min} \rightarrow 3.5^{\circ} \mathrm{C} / \mathrm{min} \rightarrow 145^{\circ} \mathrm{C}$,

Duration of Analysis: 28 min.

\subsubsection{Identification}

\section{1) Molecular Identification}

Molecular biological identification was conducted based on the study of Z.K. 
Xue et al. (2014).

\section{2) Characteristics of growth and HX-production}

Thirty $15-\mathrm{mL}$ anaerobic tubes were filled with $15 \mathrm{~mL}$ fermentation culture medium each and inoculated with the seed liquid of HX-producing bacteria at $10 \%$ proportion. The inoculated media were cultured in a vacuum incubator at $34^{\circ} \mathrm{C}$ and $0.08 \mathrm{MPa}$. Three anaerobic tubes were selected every 2 days for bacterial counts and HX analysis.

3) Screening fermentation culture media for high yields of $\mathrm{HX}$

Four fermentation culture media, as described by Z.K. Xue [12], were screened for high HX output.

\subsubsection{Optimization of Fermentation Culture Media}

\section{1) Screening of carbon source, nitrogen source, and inorganic salt}

A single-factor experiment was used to change only one nutrient for the fermentation culture medium, e.g., the sodium acetate medium mentioned in section 1.2.3 of the present paper. The amounts of carbon sources (glucose, starch, lactose, and sucrose), nitrogen sources (yeast extract, beef extract, peptone, ammonium chloride, ammonium sulfate, and urea), and inorganic salts $(\mathrm{KCl}$, $\mathrm{K}_{2} \mathrm{HPO}_{4}, \mathrm{KH}_{2} \mathrm{PO}_{4}, \mathrm{MgSO}_{4}, \mathrm{MnSO}_{4}, \mathrm{CaCO}_{3}$ ) were the same as those in the screened fermentation medium.

\section{2) Single-factor experiment}

A single-factor experiment was performed to determine the dosage range of the main components of fermentation culture medium A, e.g., glucose, yeast extracts (used instead of beef extract due to cost), $\mathrm{KCl}$, sodium acetate, and alcohol. The experimental concentration gradient was composed of two to three concentration levels that increased or decreased the concentrations of the components of the sodium acetate culture medium.

3) Optimization of fermentation culture medium by Taguchi design method

Based on the single-factor experiment, four ingredients, e.g., yeast extract, sodium acetate, ethanol, and $\mathrm{KCl}$, of the fermentation culture medium at three levels (Table 1) were selected for further optimization. A scheme for the orthogonal test (Table 2), which includes three factors and three levels, was designed using the Taguchi design method in Minitab 17.

Table 1. Factors and levels.

\begin{tabular}{ccccc}
\hline & \multicolumn{4}{c}{ Factor } \\
\cline { 2 - 5 } Level & Yeast extract & $\mathrm{KCl}$ & Sodium acetate & Ethanol \\
\cline { 2 - 5 } & & $\mathrm{g} / \mathrm{L}$ & $\mathrm{mL} / \mathrm{L}$ \\
\hline 1 & 1.5 & 1.2 & 15 & 10 \\
2 & 2.5 & 1.8 & 20 & 15 \\
3 & 3.5 & 2.4 & 25 & 20 \\
\hline
\end{tabular}


Table 2. Orthogonal test for HX yields.

\begin{tabular}{ccccc}
\hline \multirow{2}{*}{ Group } & Yeast extract & $\mathrm{KCl}$ & Sodium acetate & Ethanol \\
\cline { 2 - 5 } & & $\mathrm{g} / \mathrm{L}$ & & $\mathrm{mL} / \mathrm{L}$ \\
\hline 1 & 1.5 & 1.2 & 15 & 10 \\
2 & 1.5 & 1.8 & 20 & 15 \\
3 & 1.5 & 2.4 & 25 & 20 \\
4 & 2.5 & 1.2 & 20 & 20 \\
5 & 2.5 & 1.8 & 25 & 10 \\
6 & 2.5 & 2.4 & 15 & 15 \\
7 & 3.5 & 1.2 & 25 & 15 \\
8 & 3.5 & 1.8 & 15 & 20 \\
9 & 3.5 & 2.4 & 20 & 10 \\
\hline
\end{tabular}

The total cell number, which is represented by the optical density (OD) of 10-day fermentation broth, was detected by a spectrophotometer. The caproic acid content of the 15-day fermentation broth was detected using GC.

\section{4) Validation Experiments}

Validation experiments were performed based on the optimal formula in Section 2.2.3.3).

\subsubsection{Data Processing}

Data were processed using Excel 2007 (Microsoft Excel, USA), Minitab 17 (PA, USA), and SPSS 19 (Chicago, USA).

\section{Results and Discussion}

\subsection{GC Analysis of Fermented Broth}

The HX chromatograms of the standard material and fermentation broth were generated as shown in Figure 1.

The chromatographic peak in Figure 1 demonstrated that the GC conditions in the present study were suitable for HX detection.

The HX yields of six clones in 15-day fermented broth are shown in Table 3. The HX yields of six clones were compared with one-way ANOVA in SPSS 17. The results indicated that the HX yields of clone 5 in 15-day fermented broth was significantly higher than those of clones $1,2,3,4$, and 6 ( Sig $_{\text {tow-tailed }}=0,0,0$, $0<0.01)$. Therefore, clone 5 was selected for investigation.

\subsection{Morphological Characterization of Clone 5}

The results for the morphological characterization of clone 5 are shown in Figure 2.

Clone 5 possesses the following characteristics:

1) Colony characteristics: round or roughly round; white or yellowish; opaque or translucent; relatively uniform edges; smooth surface; colony diameter 1.5 - 2 $\mathrm{mm}$; 


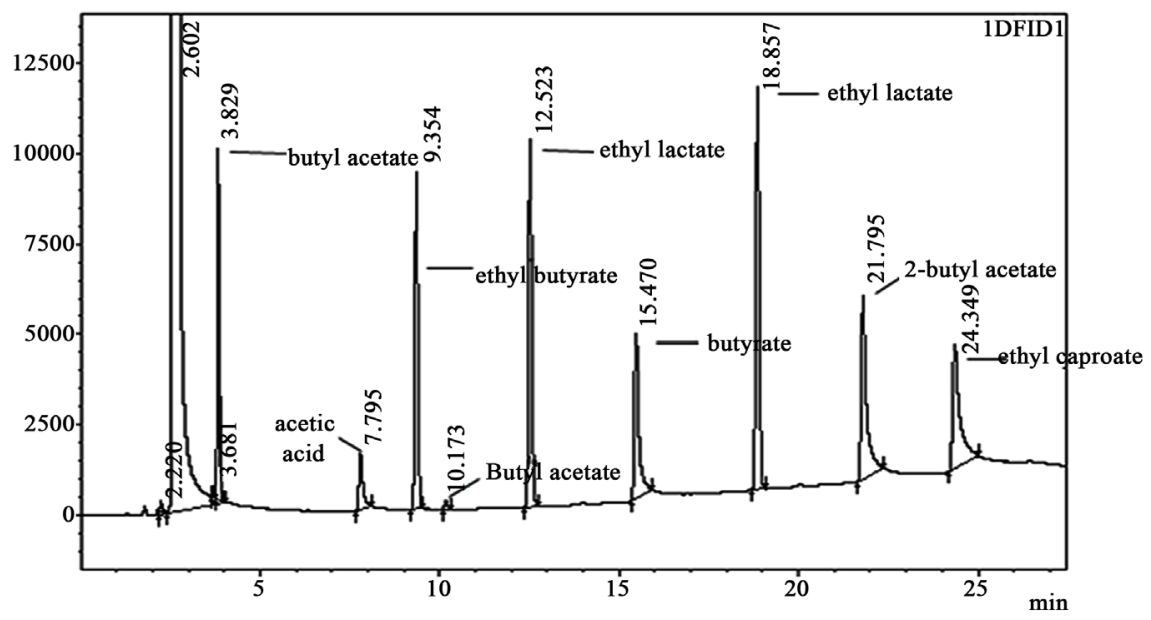

(a)

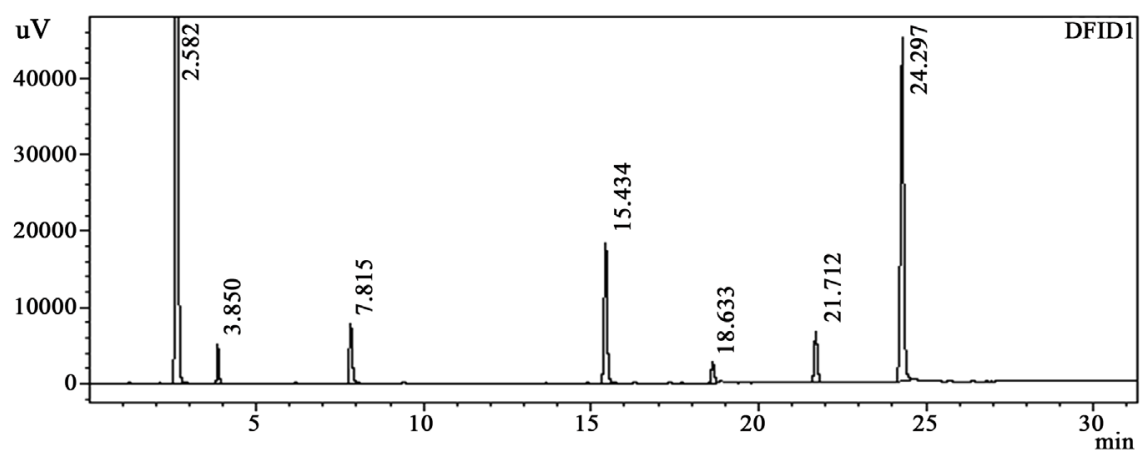

(b)

Figure 1. GC chromatograms. (a) Standard material; (b) Fermentation broth.

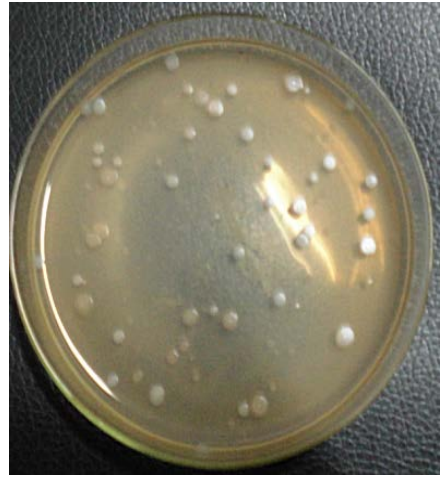

(a)

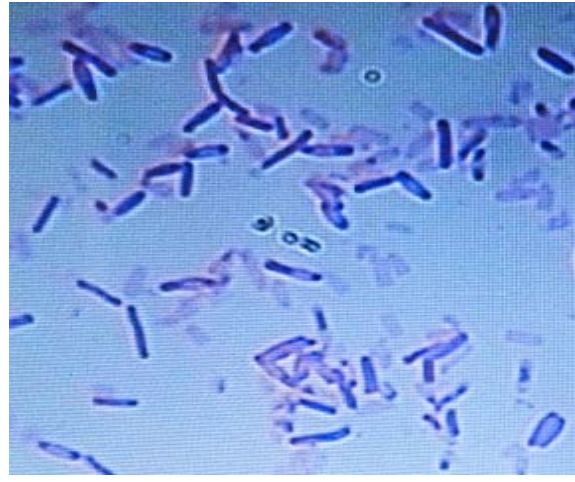

(b)

Figure 2. Morphological characterization of clone 5. (a) Colony characteristics of clone 5; (b) Bacterial cell characteristics of clone $5(10 \times 100)$.

Table 3. HX yields of six clones.

\begin{tabular}{|c|c|c|c|c|c|c|c|c|c|c|}
\hline Clones & & 1 & & 2 & & 3 & & 4 & 5 & 6 \\
\hline & 189.56 & & 234.76 & & 206.33 & & 365.89 & & 585.78 & 167.34 \\
\hline \multirow[t]{2}{*}{$\begin{array}{l}\text { HX yields } \\
(\mathrm{mg} / 100 \mathrm{~mL})\end{array}$} & 196.35 & $198.99 \pm 11$ & 286.69 & $263.1 \pm 26.29$ & 295.89 & $260.3 \pm 47.52$ & 386.78 & $383.64 \pm 16.4$ & $623.45609 .22 \pm 20.46$ & $181.55174 .43 \pm 7.11$ \\
\hline & 211.07 & & 267.84 & & 278.67 & & 398.24 & & 618.44 & 174.39 \\
\hline
\end{tabular}


2) Bacterial cell morphology: long rods;

3) Spore morphology: ellipsoidal;

4) Spore-bearing position: mostly located in the center of the cell with a small number of cells at one end;

5) Gram staining: $\mathrm{G}^{+}$.

\subsection{Molecular Identification}

The gel electrophoresis results of 16SrDNA amplified by PCR were listed in Figure 3, the sequencing results for 16SrDNA fragments is listed in Figure 4 and the taxonomic status of clone $5 \mathrm{~b}$ was shown in Figure 5.

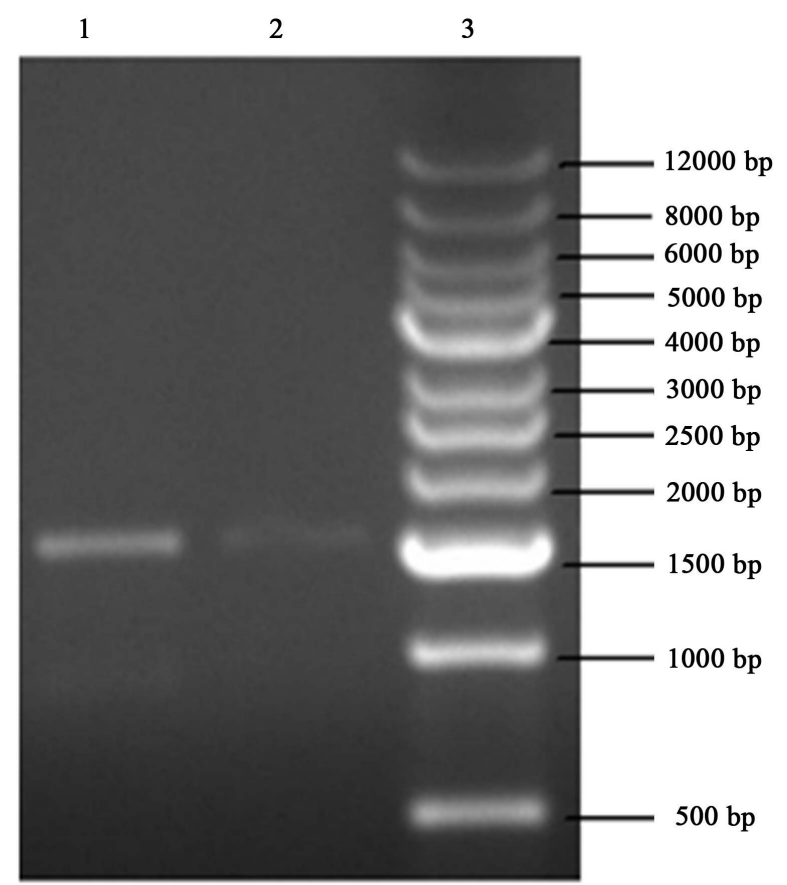

Figure 3. The $16 \mathrm{srDNA}$ of clone 5 .

TAAACATGCAAGTCGAGCGAGGAGATTCCCTTCGGGGATGAACCTAGCGGCGGACGGGTGAGTAACACGTGGGCAACCT GCCTTATAGAGGGGAATAGCCTTCCGAAAGGAAGATTAATACCGCATAACATTACATTTTCGCATGAAGAAGTAATTAAAG GAGTAATCCGCTATAAGATGGGCCCGCGGCGCATTAGCTAGTTGGTGAGGTAACGGCTCACCAAGGCGACGATGCGTAGC CGACCTGAGAGGGTGATCGGCCACATTGGGACTGAGACACGGCCCAGACTCCTACGGGAGGCAGCAGTGGGGAATATTG CACAATGGGGGAAACCCTGATGCAGCAACGCCGCGTGAGTGATGAAGGTCTTCGGATCGTAAAGCTCTGTCTTCAGGGA CGATAATGACGGTACCTGAGGAGGAAGCCACGGCTAACTACGTGCCAGCAGCCGCGGTAATACGTAGGTGGCGAGCGTT GTCCGGATTTACTGGGCGTAAAGGGAGCGTAGGCGGATTTTTAAGTGAGATGTGAAATACCCGGGCTCAACTTGGGTGCT GCATTTCAAACTGGAAGTCTAGAGTGCAGGAGAGGAGAGTGGAATTCCTAGTGTAGCGGTGAAATGCGTAGAGATTAGG AAGAACACCAGTGGCGAAGGCGACTCTCTGGACTGTAACTGACGCTGAGGCTCGAAAGCGTGGGGAGCAAACAGGATT AGATACCCTGGTAGTCCACGCCGTAAACGATGAATACTAGGTGTAGGGGTTGTCATGACCTCTGTGCCGCCGCAAACGCA TTAAGTATTCCGCCTGGGGAGTACGGTCGCAAGATTAAAACTCAAAGGAATTGACGGGGGCCCGCACAAGCAGCGGAGC ATGTGGTTTAATTTGAAGCAACGCGAAGAACCTTACCTAGACTTGACATCTCCTGAATACTCTTAATCGAGGAAGTCCCTT CGGGGACAGGAAGACAGGTGGTGCATGGTTGTCGTCAGCTCGTGTCGTGAGATGTTGGGTTAAGTCCCGCAACGAGCGC AACCCTTATTGTTAGTTGCTACCATTAAGTTGAGCACTCTAGCAAGACTGCCCGGGTTAACCGGGAGGAAGGTGGGGATG ACGTCAAATCATCATGCCCCTTATGTCTAGGGCTACACACGTGCTACAATGGCAAGTACAAAAAGATGCAATACCGCAAG GTGGAGCTAAACTTAAAAACTTGTCTCAGTTCGGATTGTAGGCTGAAACTCGCCTACATGAAGCTGGAGTTGCTAGTAAT CGCGAATCAGAATGTCGCGGTGAATACGTTCCCGGGCCTTGTACACACCGCCCGTCACACCATGAGAGTTGGCAATACCC AAAGTTCGTGAGCTAACCCGTAAGGGAGGCAGCGACCTAAGGTAGGGTCAGCGATTGGGGTGAAGTCGAACA

Figure 4. 16srDNA gene sequence. 


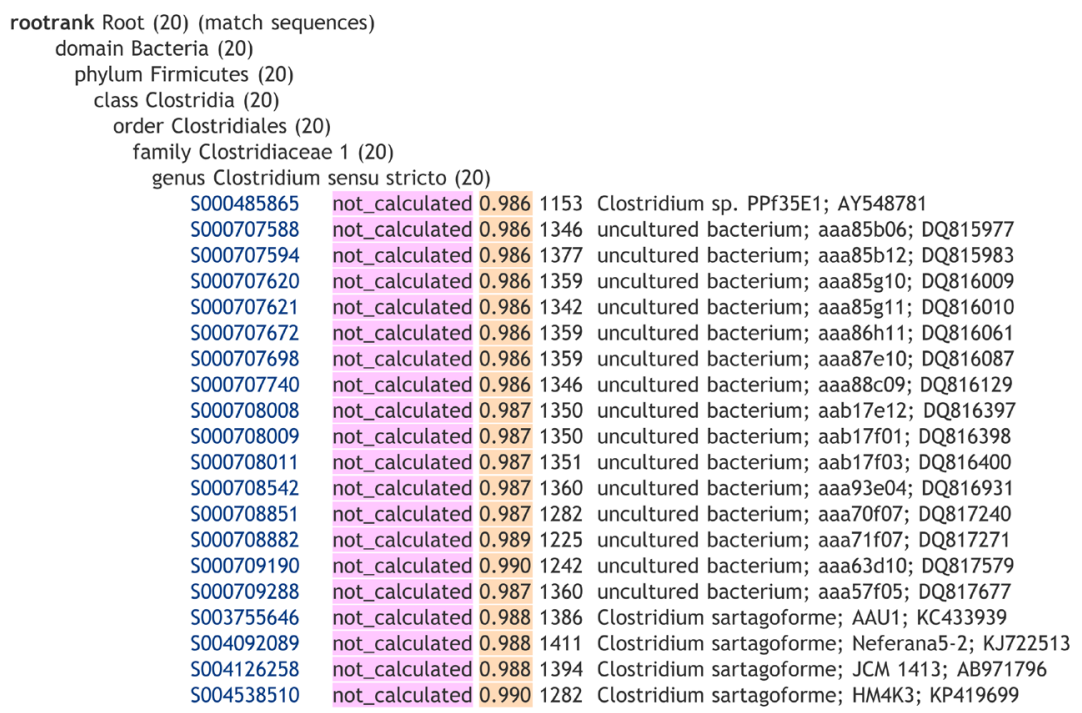

Figure 5. Taxonomic status of clone 5.

The sequence of the 16srDNA primer amplification product in Figure 4 was $1427 \mathrm{bp}$ long, the aligned results in RDP (http://rdp.cme.msu.edu/) revealed that clone 5 had a $99 \%$ sequence similarity to that of Clostridium sartagoneforme (Figure 5).

This is the first study to discover that $C$. sartagoneforme produces HX in CSFL pit mud.

\subsection{Growth Curve and HX-Production}

The results for HX-producing bacterial counts and HX contents are shown in Figure 6.

By analyzing the curves in Figure 6, the following conclusions were drawn:

1) Based on the bacterial growth curve, the initial lag period lasted for 4 days. The total number of bacterial cells (TNBC) negligibly increased during this period. The logarithmic growth phase occurred during the sixth to eighth days of fermentation. The TNBC increased the fastest during this period. The stationary phase occurred on the eighth to tenth days of fermentation. The TNBC negligibly increased during this period. Bacterial decay occurred from the 10th day to the end of fermentation. The highest TNBC was $53.72 \pm 1.32\left(\times 10^{7} \mathrm{cfu} / \mathrm{mL}\right)$ and was observed on the 10th day.

2) Given that the fastest increase in HX production occurred during the eight to the 60th day of fermentation, the optimal fermentation period for HX production is 16 days.

\subsection{Screening of HX-Producing Fermentation Culture Medium}

The results of GC analysis for HX yield in the four candidate fermentation culture media are shown in Table 4.

The results in Table 4 revealed that the highest HX yield was in fermentation culture medium A. Further analysis by one-way ANOVA indicated that HX 


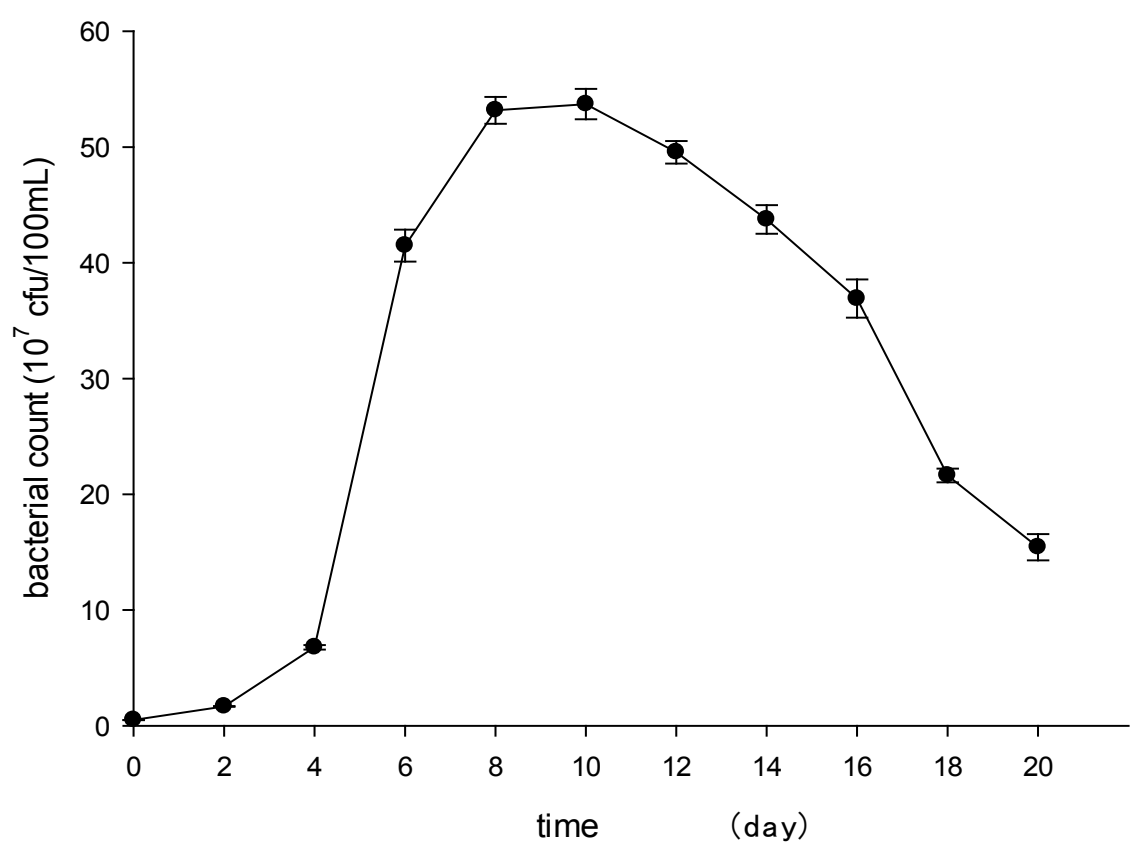

(a)

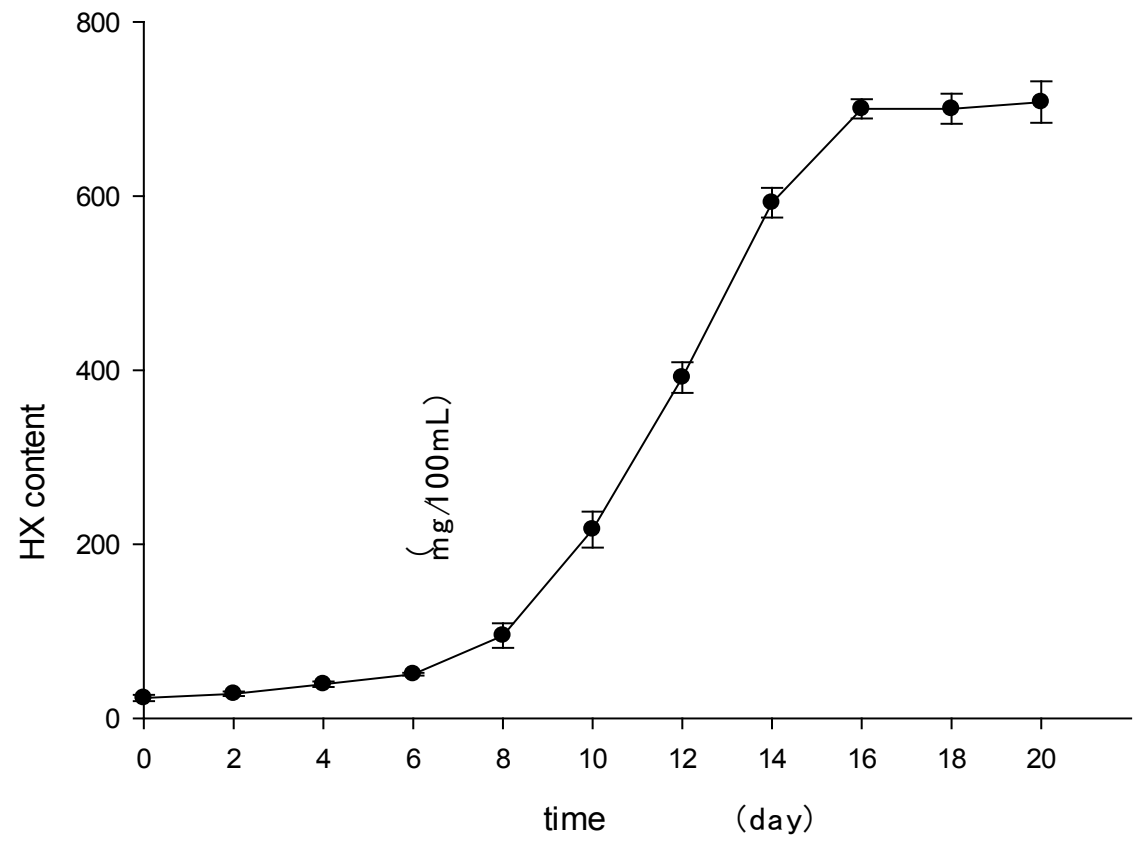

(b)

Figure 6. The growth and HX-production curves. (a) The growth curve; (b) The HX-production curve.

Table 4. X yields in four candidate fermentation culture media.

\begin{tabular}{|c|c|c|c|c|c|c|c|c|}
\hline Medium & & A & & B & & $\mathrm{C}$ & & $\mathrm{D}$ \\
\hline \multirow{3}{*}{$\begin{array}{l}\text { HX yields } \\
(\mathrm{mg} / 100 \mathrm{~mL})\end{array}$} & 788.726 & & 189.110 & & 194.150 & & 374.65 & \\
\hline & 814.352 & $800.85 \pm 12.87$ & 204.496 & $196.87 \pm 7.69$ & 203.847 & $205.54 \pm 12.32$ & 397.54 & $391.99 \pm 15.34$ \\
\hline & 799.46 & & 196.991 & & 218.617 & & 403.79 & \\
\hline
\end{tabular}


yield in fermentation culture medium A was significantly higher than those in fermentation culture media $\mathrm{B}, \mathrm{C}$, and $\mathrm{D}$. Moreover, HX yield in fermentation culture medium A was higher than those in fermentation culture media B,C, and D by $4.07,3.90$, and 2.04 times, respectively.

\subsection{Screening for Carbon Source, Nitrogen Source, and Inorganic Salt}

HX yields in fermentation liquids with various carbon sources, nitrogen sources, and inorganic salts are shown in Figure 7.

The following conclusions were drawn from the screening results for the optimal carbon source, nitrogen source, and inorganic salt for fermentation culture medium A:

1) Among the four carbon sources, the highest HX output (Figure 7(a)) was achieved with $1 \mathrm{~g} / \mathrm{L}$ glucose as the carbon source. ANOVA analysis revealed that HX output with glucose as the carbon source was significantly higher $(\mathrm{P}<0.01)$ than those with lactose, sucrose, or starch as carbon sources.

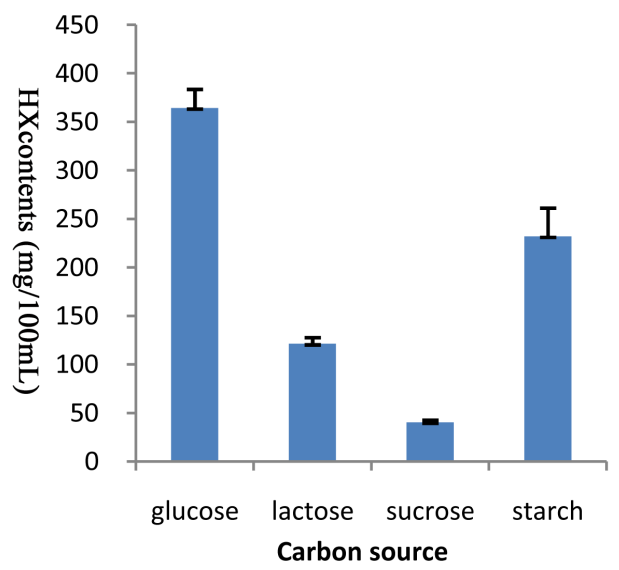

(a)

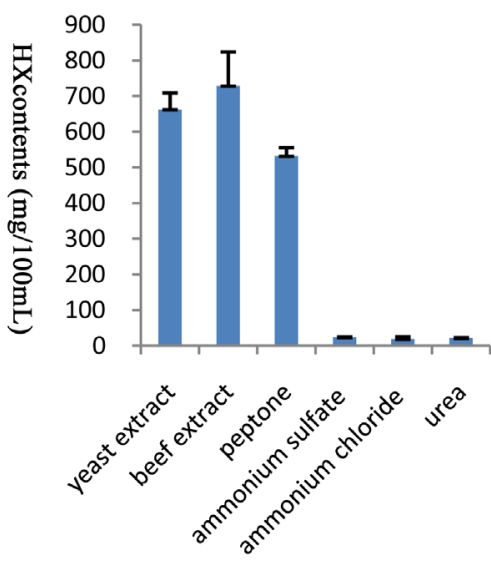

Nitrogen source

(b)

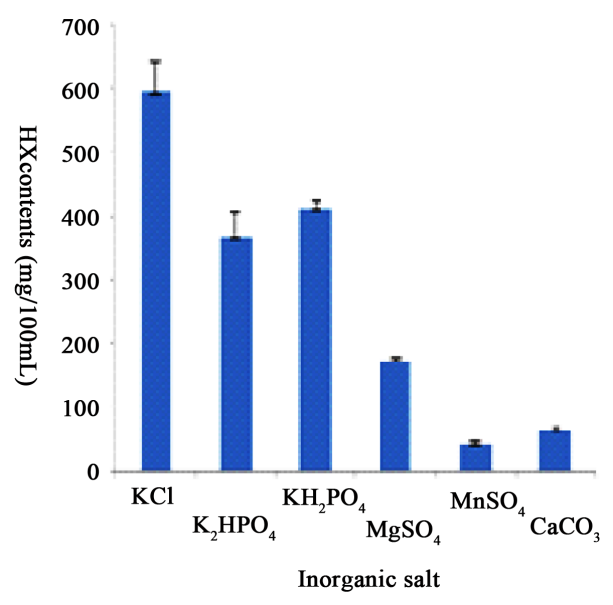

(c)

Figure 7. Screening results for carbon source (a); nitrogen source (b); and inorganic salt (c). 
2) Among the six nitrogen sources, the highest HX output (Figure 7(b)) was achieved with $2.5 \mathrm{~g} / \mathrm{L}$ beef extract as then nitrogen source. ANOVA analysis revealed that HX output in the fermentation liquid with beef extract as the nitrogen source was significantly higher than those with $(P=0.048)$, ammonium sulfate, ammonium chloride, and urea $(\mathrm{P}<0.01)$ as nitrogen sources. HX yields with yeast or beef extracts as nitrogen sources were not significantly different $(P$ $=0.23$ ). Therefore, yeast extract was used as the nitrogen source for further optimization because of economic reasons.

3) Among the inorganic salt sources, the highest HX output (Figure 7(c)) was achieved with $1.8 \mathrm{~g} / \mathrm{L}$ KCL.ANOVA analysis revealed that the HX output with KCL was significantly higher than all the others $(P<0.01)$.

Therefore, the optimal carbon source, nitrogen source, and inorganic salt for $\mathrm{HX}$ production were glucose, beef extract, and $\mathrm{KCl}$.

\subsection{Single-Factor Experiment}

The HX contents in the 15-day fermentation liquid of the single-factor experiment are shown in Figure 8.

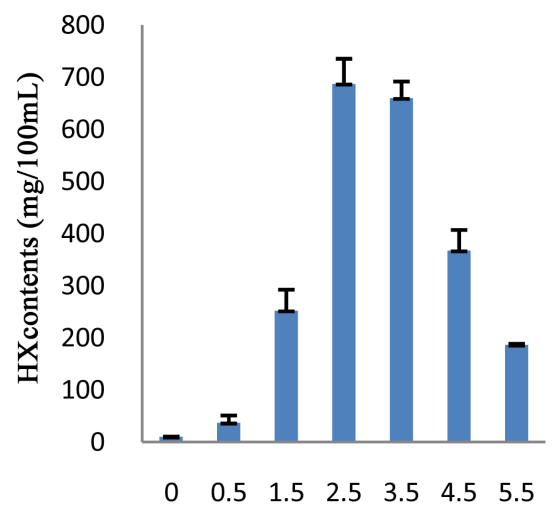

Concentration of yeat extrac $\mathrm{t}(\mathrm{g} / \mathrm{L})$

(a)

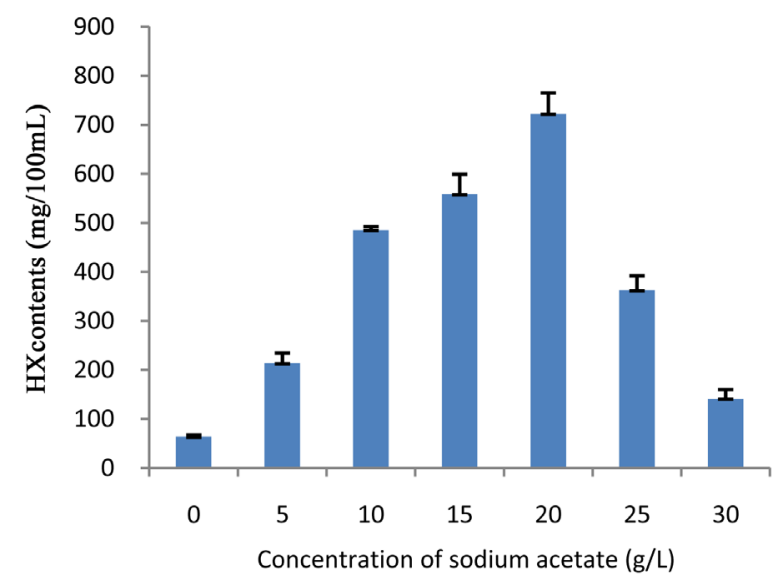

(d)

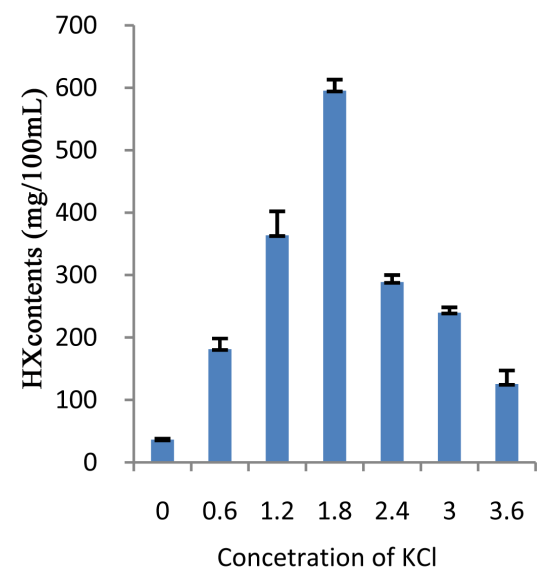

(b)

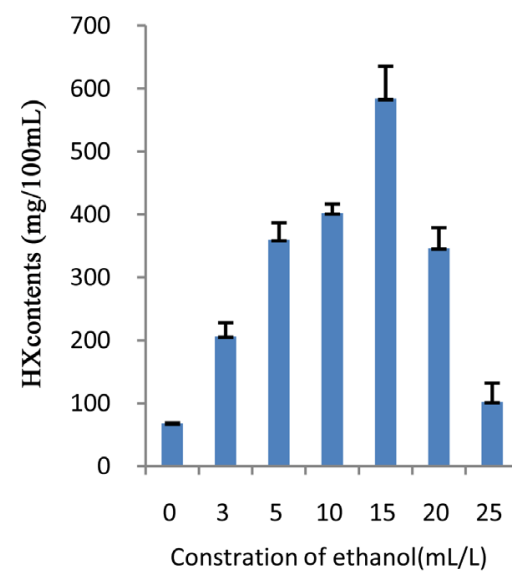

(c)

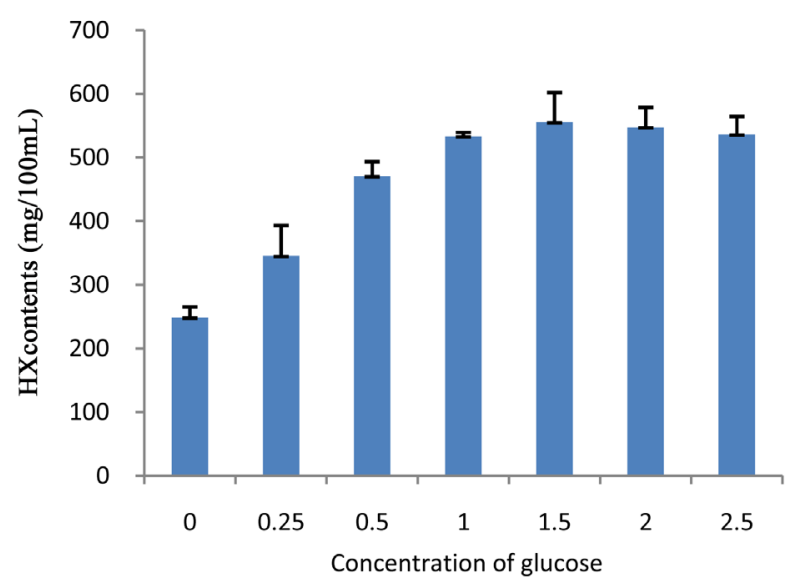

(e)

Figure 8. Results of single-factor experiment. 
The optimal concentration for each ingredient of the fermentation culture medium in $\mathrm{HX}$ production with strain $\mathrm{K} 3$ was $2.5 \mathrm{~g} / \mathrm{L}$ yeast extract, $1.8 \mathrm{~g} / \mathrm{L} \mathrm{KCl}$, $15 \mathrm{~mL} / \mathrm{L}$ ethanol, $20 \mathrm{~g} / \mathrm{L}$ sodium acetate, and $1.5 \mathrm{~g} / \mathrm{L}$ glucose. The influence of each individual factor was calculated by subtracting the zero concentration from the highest HX production. Comparing the effects of individual factors revealed that sodium acetate was the most effective factor of HX production, whereas glucose was the most ineffective. Therefore, glucose was excluded from further optimization.

\subsection{Total Cell Number of Fermentation Broth by Taguchi Design Method}

A spectrophotometer was used to detect the total cell number, which was represented by OD, in 10-day fermentation broth that was optimized via the Taguchi design method. The results are shown in Figure 9.

The OD in Figure 9 demonstrated that group 1 had the highest cell number among all experimental groups. Comparing the OD in all the experimental groups revealed that the OD values of groups $1,2,6$, and 9 were not significantly different $(P>0.05)$. The OD values of these groups, however, are significantly higher than those of other groups $(\mathrm{P}<0.05)$.

\subsection{Optimization of HX Production by Taguchi Design Method}

The GC detection results of the 16-day fermentation liquid are shown in Table 5.

Minitab 17 (Minitab Inc.; State College, PA) software was used to analyze the experimental results in Table 5 to obtain the response means of HX content, response means of $\mathrm{SN}$ ratios, mean main effect diagram, and the $\mathrm{SN}$ ratio of the main effect diagram. The location, dispersion, and adjustment factors were analytically determined to obtain the optimal factor and level combination for the fermentation medium [14]. The results were showed in Table 6, Table 7, Figure 10, and Figure 11.

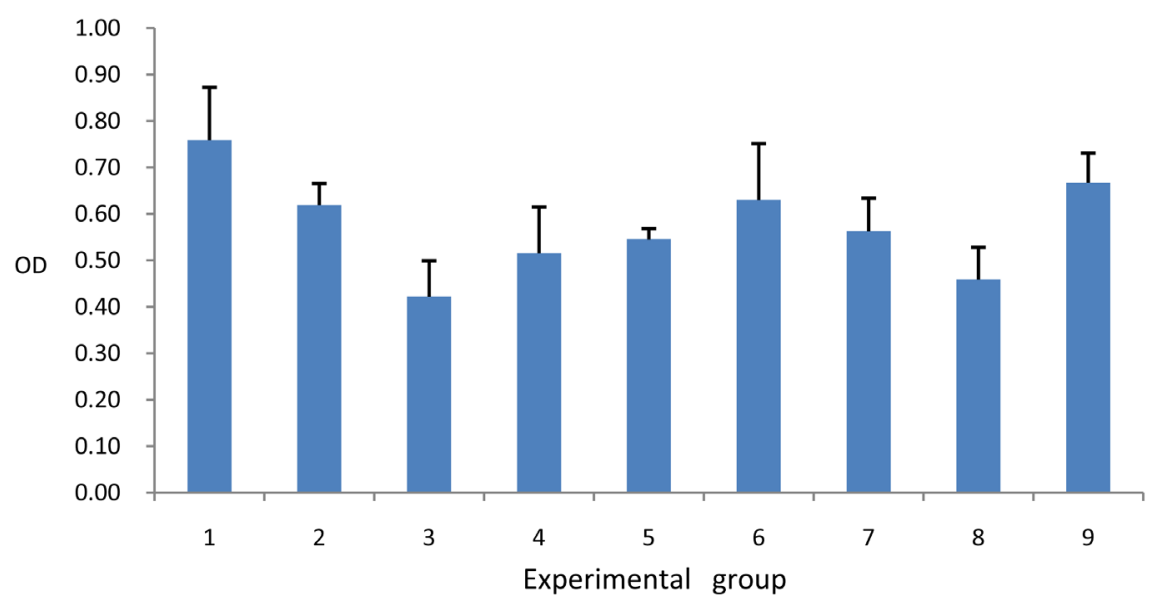

Figure 9. OD (optical density) of 10-day fermentation broth. 


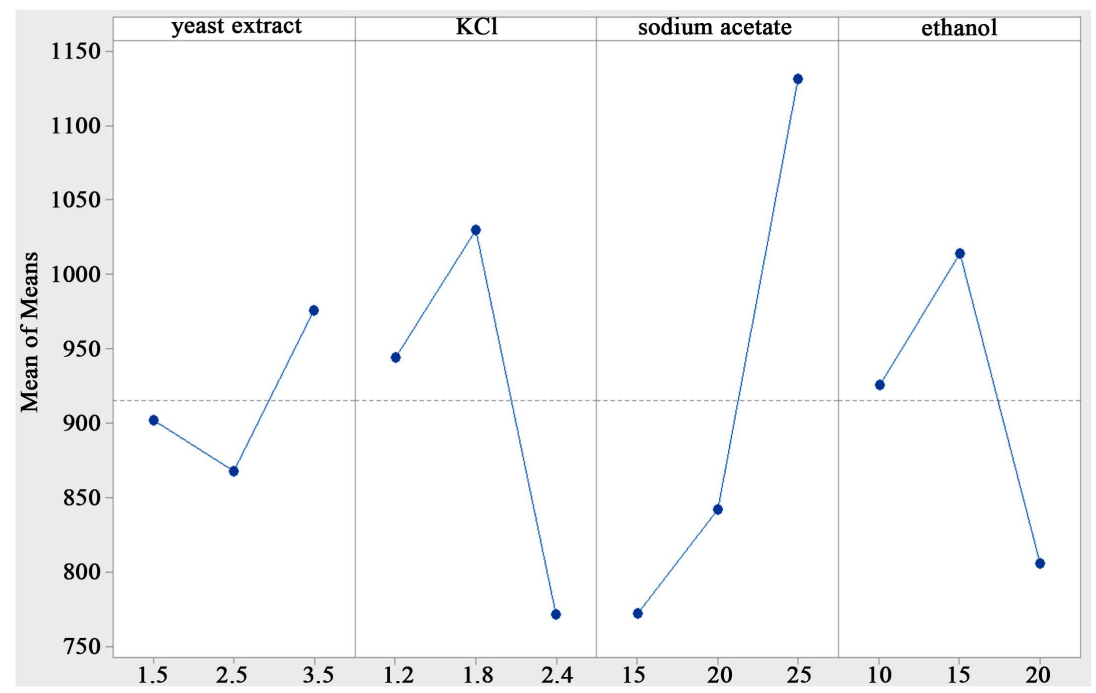

Figure 10. Main effects diagram of each factor for HX content.

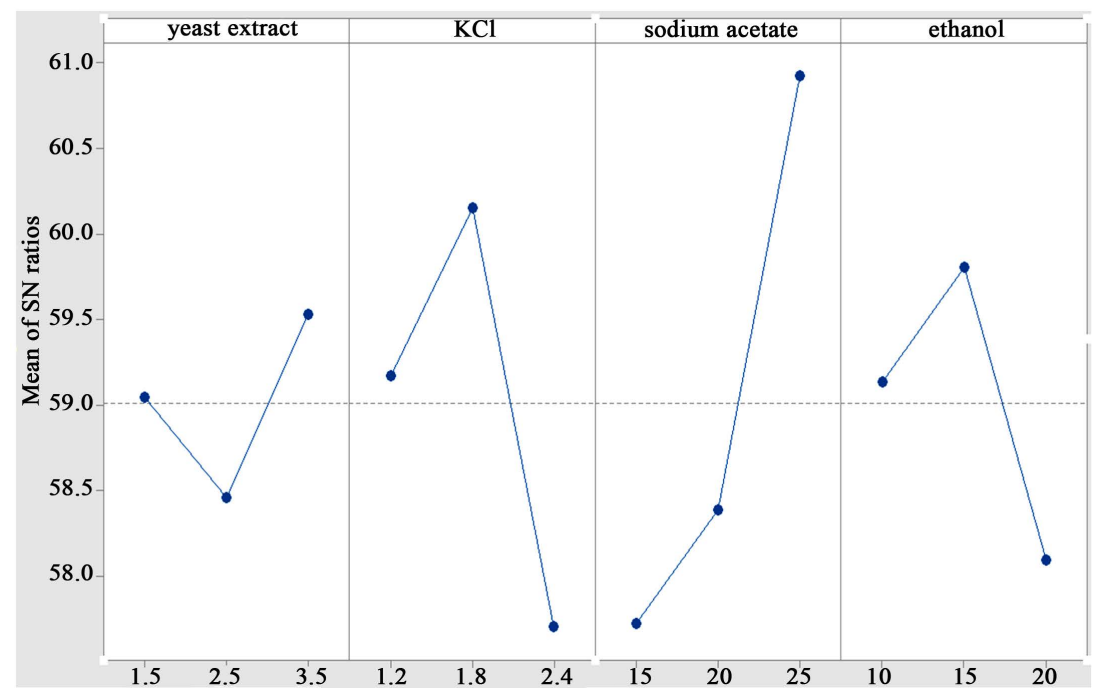

Figure 11. Main effects diagram of each factor for SN ratios.

Table 5. The HX content of the Taguchi design.

\begin{tabular}{|c|c|c|c|c|c|c|c|c|}
\hline \multirow{2}{*}{$\begin{array}{c}\text { Test } \\
\text { number }\end{array}$} & \multicolumn{4}{|c|}{ Factors } & \multicolumn{3}{|c|}{$\mathrm{HX}$ content $(\mathrm{mg} / 100 \mathrm{~mL})$} & \multirow{2}{*}{$\begin{array}{c}\mathrm{Y} \\
(\mathrm{mg} / 100 \mathrm{~mL})\end{array}$} \\
\hline & $\begin{array}{c}\text { Yeast } \\
\text { extract }(\mathrm{g} / \mathrm{L})\end{array}$ & $\begin{array}{l}\mathrm{KCl} \\
(\mathrm{g} / \mathrm{L})\end{array}$ & $\begin{array}{c}\text { Sodium } \\
\text { acetate }(\mathrm{g} / \mathrm{L})\end{array}$ & $\begin{array}{c}\text { Ethanol } \\
(\mathrm{mL} / \mathrm{L})\end{array}$ & 1 & 2 & 3 & \\
\hline 1 & 1.5 & 1.2 & 15 & 10 & 782 & 795 & 820 & 799 \\
\hline 2 & 1.5 & 1.8 & 20 & 15 & 1050 & 1026 & 1050 & 1042 \\
\hline 3 & 1.5 & 2.4 & 25 & 20 & 838 & 870 & 888 & 865.33 \\
\hline 4 & 2.5 & 1.2 & 20 & 20 & 717 & 730 & 697 & 714.67 \\
\hline 5 & 2.5 & 1.8 & 25 & 10 & 1169 & 1190 & 1269 & 1209.33 \\
\hline 6 & 2.5 & 2.4 & 15 & 15 & 671 & 680 & 691 & 680.67 \\
\hline 7 & 3.5 & 1.2 & 25 & 15 & 1283 & 1295 & 1383 & 1320.33 \\
\hline 8 & 3.5 & 1.8 & 15 & 20 & 828 & 830 & 858 & 838.67 \\
\hline 9 & 3.5 & 2.4 & 20 & 10 & 759 & 770 & 780 & 769.67 \\
\hline
\end{tabular}


Table 6. Response means for HX content.

\begin{tabular}{ccccc}
\hline \multirow{2}{*}{ Level } & \multicolumn{4}{c}{ Factors } \\
\cline { 2 - 5 } & Yeast extract & $\mathrm{KCl}$ & Sodium acetate & Ethanol \\
\hline 1 & 902.1 & 944.7 & 772.8 & 926.0 \\
2 & 868.2 & 1030.0 & 842.1 & 1014.3 \\
3 & 976.2 & 771.9 & 1131.7 & 806.2 \\
Delta & 108.0 & 258.1 & 358.9 & 208.1 \\
Rank & 4 & 2 & 1 & 3 \\
\hline
\end{tabular}

Table 7. Response means for signal-to-noise ratios.

\begin{tabular}{ccccc}
\hline \multirow{2}{*}{ Level } & \multicolumn{4}{c}{ Factors } \\
\cline { 2 - 5 } & Yeast extract & $\mathrm{KCl}$ & Sodium acetate & Ethanol \\
\hline 1 & 59.05 & 59.18 & 57.73 & 59.14 \\
2 & 58.46 & 60.16 & 58.39 & 59.81 \\
3 & 59.54 & 57.71 & 60.94 & 58.10 \\
Delta & 1.07 & 2.45 & 3.21 & 1.71 \\
Rank & 4 & 2 & 1 & 3 \\
\hline
\end{tabular}

To identify the factors that significantly influence the mean value, the mean variance of the experimental results was analyzed further. The results are shown in Table 8.

Based on the analysis of response for the means of HX content (Table 6); response means for signal-to-noise ratios (SNR) (Table 7); main effect diagram for means of HX content (Figure 10); main effects diagram for SN ratios of each factor (Figure 11); results of variance analysis (Table 8), and with caproic acid yields as the index, yeast extract, $\mathrm{KCl}$, sodium acetate, and ethanol all significantly influence HX content. The relative importance of these factors was as follows: sodium acetate $>\mathrm{KCl}>$ ethanol $>$ yeast extract. The optimal factor and level combination for HX output was level 3 yeast extract, level $2 \mathrm{KCl}$, level 3 sodium acetate, and level 2 ethanol. Specifically, the optimal formula for maximum HX output was $3.5 \mathrm{~g} / \mathrm{L}$ yeast extract, $1.8 \mathrm{~g} / \mathrm{L} \mathrm{KCl}, 25 \mathrm{~g} / \mathrm{L}$ sodium acetate, and 15 $\mathrm{mL} / \mathrm{L}$ ethanol. The predicted HX yield using this formula was 2112.346 $\mathrm{mg} / 100 \mathrm{~mL}$.

\subsection{Validation Experiment}

The mean of HX yields in the validation experiment was $2018.29 \pm 46.37$ $\mathrm{mg} / 100 \mathrm{~mL}$ and the SNR was $8.78 \mathrm{~dB}$. The results demonstrated that the HX yields in the validation experiment were close to the predicted HX yield, thus indicating that this optimization scheme can achieve the desired effect and has good stability. 
Table 8. Analysis of variance of mean value.

\begin{tabular}{cccccc}
\hline Source & DF & AdjSS & AdjMS & F-value & P-value \\
\hline Yeast extract & 2 & 47115 & 23557 & 233.07 & 0 \\
KCl & 2 & 288283 & 144142 & 1426.10 & 0 \\
Sodium acetate & 2 & 592738 & 296369 & 2932.20 & 0 \\
Ethanol & 2 & 173352 & 86676 & 857.55 & 0 \\
Error & 18 & 1819 & 101 & & \\
Total & 26 & 1103307 & & & \\
\hline
\end{tabular}

\section{Conclusion}

In the present study, an efficient HX-producing strain was isolated from 280 -year-old pit mud. This strain yielded $609.22 \pm 20.46 \mathrm{mg} / 100 \mathrm{~mL}$ HX. Morphological identification indicated that the strain possessed the typical characteristics of HX-producing strains, such as $\mathrm{G}^{+}$and spore formation. Bioinformatics analysis revealed that the strain shared $99.1 \%$ sequence similarity with $C$. sartagoneforme. Meanwhile, the four candidate fermentation culture media A, B, C, and $\mathrm{D}$ were tested on the basis of maximum caproic acid yield. Single-factor experiments were performed based on the selected fermentation culture medium A. The results of the single-factor tests showed that the maximum HX yields in 15-day-old fermentation broth were obtained with culture medium A that contained $2.5 \mathrm{~g} / \mathrm{L}$ yeast extract, $1.8 \mathrm{~g} / \mathrm{L} \mathrm{KCl}, 20 \mathrm{~g} / \mathrm{L}$ sodium acetate, and $15 \mathrm{~mL} / \mathrm{L}$ ethanol. A further orthogonal experiment was designed using Taguchi methods in Minitab 17. The results demonstrated that the optimum formula for maximum HX output was $3.5 \mathrm{~g} / \mathrm{L}$ yeast extract, $1.8 \mathrm{~g} / \mathrm{L} \mathrm{KCl,} 25 \mathrm{~g} / \mathrm{L}$ sodium acetate, and $15 \mathrm{~mL} / \mathrm{L}$ ethanol. The validation experiment had a maximum HX yield of $2018.29 \pm 46.37 \mathrm{mg} / 100 \mathrm{~mL}$.

\section{Acknowledgements}

This research was financially supported by the Science and Technology Support Plan Project of Sichuan Province (No. 2014FZ0018), the Social Development Project of Luzhou Municipal Science and Technology Bureau (2017-S-45 (3/3)), and the Key Fund Project of Sichuan Provincial Department of Education (18ZA025).

\section{Conflicts of Interest}

The authors declare that they have no competing interests.

\section{References}

[1] Zhang, F.L. (2008) Utilization of Caproic Acid Bacteria Pit Mud for Fermentation to Improve the Yield of Kouzi Liquor. Liquor-Making Science \& Technology, 22, 823-830.

[2] Yang, G.R. and Tang, Y.T. (2010) Culture of Manmade Pit Mud \& Its Application. 
Liquor Making, 37, 25-27.

[3] Xia, P.Y., Xun-Liang, S.U. and Xi-Wang, X.U. (2005) Application of TH-AADY in the Production of Manmade Pit Mud. Liquor-Making Science \& Technology, 136, 37-38.

[4] Barros, D.P.C.D., Pinto, F., Fonseca, L.P., Cabral, J.M.S. and Lemos, F. (2014) Kinetic Model for the Esterification of Ethyl Caproate for Reaction Optimization. Journal of Molecular Catalysis B: Enzymatic, 101, 16-22. https://doi.org/10.1016/j.molcatb.2013.12.012

[5] Tan, Z., Bao, X., Du, H., Chen, Q., Han, X. and Li, J. (2014) Synthesis of Ethyl Caproate in Dbsa/Cyclohexane/Water Microemulsion System. Journal of Chinese Institute of Food Science \& Technology, 14, 140-144.

[6] Sun, B.G., Sun, J.Y., Gong, L.L. and Sun, X.T. (2016) Perspectives on the Long-Term Development Trend and Research Emphasis of Chinese Baijiu (Chinese liquor). Journal of Light Industry, 31, 6-11.

[7] Liu M., Tang, Y., Zhao, K., Ren, D., Yao, W. and Tian, X. (2015) Analysis of Action Bacteria Community and Diversity in the Pit Mud of Chinese Luzhou-Flavour Liquor. Acta Ecologica Sinica, 95, 2729-2735.

[8] Fan, W.L. and Xu, Y. (2014) Current Practice and Future Trends of Aroma and Flavor of Chinese Liquor (Baijiu). Journal of Food Safety and Food Quality, 2014, 3073-3078.

[9] Chen, Y., Luo, W., Rui, G., Xue, X., Guan, X., Song, L., et al. (2016) Improved Ethyl Caproate Production of Chinese Liquor Yeast by Overexpressing Fatty Acid Synthesis Genes with Opi1, Deletion. Journal of Industrial Microbiology \& Biotechnology, 43, 1-10. https://doi.org/10.1007/s10295-016-1795-x

[10] Cui, H.H. and Cui, J.J. (2014) Isolation and Purification of Caproic Acid Bacteria from Shilixiang Aged Pit Mud and Study of Its Expand Training and Application. Liquor Making, 41, 39-41.

[11] Hu, X.L., Du, H. and Xu, Y. (2015) Identification and Quantification of the Caproic Acid-Producing Bacterium Clostridium Kluyveri, in the Fermentation of Pit Mud Used for Chinese Strong-Aroma Type Liquor Production. International Journal of Food Microbiology, 214, 116-122. https://doi.org/10.1016/j.ijfoodmicro.2015.07.032

[12] Xue, Z.K. (2014) Isolation and Identification of a Caproic Acid-Producing k-1 Strain from Luzhou-Liquor Pit Mud. Journal of Proteomics, 74, 2510-2521.

[13] Huang, Z., Wei, C., Bian, M., Zong, X., Luo, H. and Zheng, R. (2012) Isolation and Identification of Two Bacteria Strains from Pit Mud of Luzhou-Flavor Liquor. Liquor-Making Science \& Technology, 221, 36-38.

[14] Aminuddin, N.A.B., Garzareyes, J.A., Kumar, V., Antony, J. and Rochalona, L. (2016) An Analysis of Managerial Factors Affecting the Implementation and Use of Overall Equipment Effectiveness. International Journal of Production Research, 54, 4430-4447. https://doi.org/10.1080/00207543.2015.1055849 